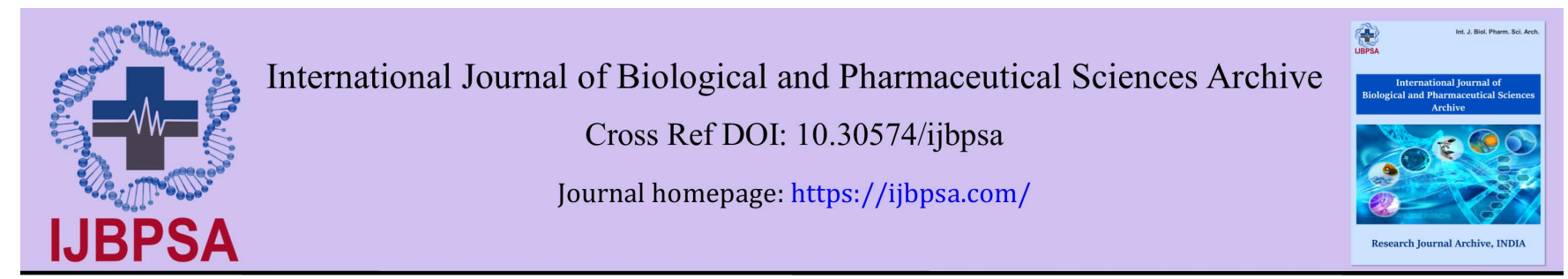

(REVIEW ARTICLE)

\title{
Oral aspects related to SARS-CoV-2
}

\author{
Akram Belmehdi ${ }^{1, *}$ and Saliha Chbicheb ${ }^{2}$ \\ ${ }_{1}^{1}$ Specialist in the department of Oral surgery, Faculty of Dentistry, Mohammed V University in Rabat, Morocco. \\ 2 Professor and head of the department of Oral surgery, Faculty of Dentistry, Mohammed V University in Rabat, Morocco.
}

International Journal of Biological and Pharmaceutical Sciences Archive, 2021, 01(02), 153-160

Publication history: Received on 13 April 2021; revised on 19 May 2021; accepted on 23 May 2021

Article DOI: https://doi.org/10.30574/ijbpsa.2021.1.2.0038

\begin{abstract}
The pandemic of coronavirus disease (COVID-19) is considered as the biggest global health crisis for the world since the Spanish flu, also known as the 1918 flu pandemic. Driven by the SARS-CoV-2 novel coronavirus infection, the rapid spread of this disease and the related pneumonia COVID-19 are a challenge for healthcare systems in over the world, and it is a constantly evolving situation with new symptoms and prognostic factors.

SARS-CoV-2 has lately been detected in infected patient's oral cavity; the COVID-19 outbreak is an alert that all dental and other health professionals must be vigilant in defending against the infectious disease spread.

This review summarizes an update from current medical literature about the relationship between oral cavity and coronavirus disease by presenting some oral aspects which was detected in infected patients such as the oral lesions related to this virus and its therapeutic protocol, taste disorders and also the diagnostic value of saliva for SARS-CoV-2.
\end{abstract}

Keywords: SARS-CoV-2; Covid-19; Oral manifestations; Saliva; Gustatory disorders

\section{Introduction}

The present outbreak of the 2019 coronavirus strain (COVID-19) constitutes a worldwide public health emergency [1]. The novel coronavirus, severe acute respiratory syndrome coronavirus 2 (SARS-CoV-2), discovered in December 2019 in China, is a highly contagious enveloped single stranded RNA virus that results in a life-threatening pulmonary illness known as COVID-19 [2,3].

On January 30, 2020, the rampant spread of SARS-CoV-2 and its associated disease was declared by World Health Organization (WHO) as a public health emergency with a currently known overall mortality rate to be as high as $3.4 \%$ $[3,4]$. On February 11, 2020, WHO used the term COVID-19 to name the latest strain of coronavirus $[4,5,6]$. In humans, six different coronavirus have been identified: HCoV-0C43, HCoV-229E, HCoV-NL63, HKU1, the Middle East respiratory syndrome (MERS)-CoV and (SARS)-CoV [7, 8]. Although the latter virus became widely discussed recently, the first human coronaviruses were isolated for the first time in 1937 [9]. The choice of coronavirus denomination was due to its microscopic aspect resembling crown-like spikes on its surface and the main host receptor for humans seems to be the Angiotensin-Converting Enzyme 2 (ACE2) [8, 10].

Patients with COVID-19 usually experienced fever and dry cough, while some also had shortness of breath, fatigue, and other atypical symptoms such as muscle pain, confusion, headache, sore throat, vomiting and diarrhea. Reduced sense of smell (hyposmia to anosmia), and abnormal taste sensation (ageusia and hypogeusia) have also been reported [11,

\footnotetext{
* Corresponding author: Akram Belmehdi

Specialist in the Oral surgery department, Faculty of Dentistry, Mohammed V University in Rabat, Morocco.
}

Copyright (c) 2021 Author(s) retain the copyright of this article. This article is published under the terms of the Creative Commons Attribution Liscense 4.0. 
12]. Among patients who underwent chest computed tomography, most showed bilateral pneumonia, with groundglass opacity and bilateral patchy shadows being the most common patterns $[12,13]$.

Some specific aspects and characteristics of the virus remain yet unknown given the novelty of SARS-CoV-2. The COVID19 outbreak serves as both a reminder and an opportunity to assist. Considering that SARS-CoV-2 was recently identified in saliva of infected patients $[14,15]$, the COVID-19 outbreak is a reminder that dental/oral and other health professionals must always be rigorous and accurate in protecting against the spread of infectious disease, and it provides a chance to determine if a non-invasive saliva diagnostic for COVID-19 could assist in detecting such viruses and reducing the spread [15].

SARS-CoV-2 human-to-human transmission occurs firstly between family members, including relatives and friends who have intimate contact with infected or asymptomatic patients or carriers. Several potential scenarios of SARS-CoV-2 transmission have been described. The transmission via contact with droplets from talking, coughing, sneezing (related to human respiratory activities), and aerosols generated during clinical procedures is expected, as it would be for other respiratory infections. The origin of droplets can be nasopharyngeal or oropharyngeal, normally associated with saliva. Larger droplets could contribute to viral transmission to subjects nearby, and, on the other side, the long-distance transmission is possible with smaller droplets infected with air-suspended viral particles [16].

Furthermore, the presence of SARS-CoV-2 in swabs from fecal and blood samples has been identified, indicating the possibility of multiple routes of infection [17]. The laboratory diagnostic test is among the priorities to facilitate public health interventions. It should be performed using nasopharyngeal, oropharyngeal, and blood samples [15]. In acute respiratory infections; RT-PCR is routinely used to detect viruses caused by respiratory secretions. During international health emergencies, the viability of real-time detection of the virus by real-time RT-PCR has been demonstrated through coordination between public laboratories and universities $[8,18]$.

The oral cavity, as a secondary external opening for the respiratory tract, presents a potentially huge COVID-19 infectious vulnerability risk and brought up a proof for the possible presence of some oral aspects of this disease. Moreover, due to the characteristics of dental settings, the risk of cross infection may be high between dental/oral practitioners and patients.

\section{Methods}

This literature review was performed from recent publications related to the COVID-19 pandemic to provide a more comprehensive understanding of the effects of SARS-CoV-2 on oral cavity; such as the possible role of saliva in early detection and transmission, the taste disorders and oral lesions. Unfortunately, there are limited evidence-based data available, as, which is understandable clinicians and scientists must focus on life-saving aspects.

\section{Discussion}

\subsection{The role of saliva in COVID-19 test}

Saliva is a complex mixture comprising of proteins, enzymes, hormones, antibodies, cytokines and antimicrobial constituents [19]. Entry process of these constituents from the blood into the saliva is by transcellular, passive intracellular diffusion and active transport, or paracellular routes by extracellular ultrafiltration within the salivary glands or through the gingival crevice. Recent technologies have reported a numbers of medically important salivary biomarkers for different disease conditions including cancer, viral, autoimmune, bacterial, cardiovascular and metabolic diseases $[20,21,22]$.

As the oral cavity is an entrance and an outlet of body, saliva may have a role in early diagnosis and close contact transmission in infectious diseases. SARS-CoV-2 is associated with human-to-human transmission and was recently detected in the saliva of infected patients [23].

Detection of some virus strains in saliva has been reported 29 days after infection [24, 25], which indicated that a rapidly non-invasive platform; to differentiate the biomarkers using saliva, could enhance disease detection [15, 26].

There is a suggestion of a minimum three different pathways for SARS-CoV-2 to present in saliva: firstly, from SARSCoV-2 in the lower and upper respiratory tract $[27,28]$ that enters the oral cavity together with the liquid droplets frequently exchanged by these organs. Secondly, SARS-CoV-2 present in the blood can access the mouth via crevicular 
fluid, an oral cavity-specific exudate that contains local proteins derived from extracellular matrix and serum-derived proteins [15]. Finally, another way for SARS-CoV-2 to occur in the oral cavity is by major and minor salivary gland infection, with subsequent release of particles in saliva via salivary ducts. It is essential to point out that salivary gland epithelial cells can be infected by SARS-CoV a short time after infection in rhesus macaques, suggesting that salivary gland cells could be a pivotal source of this virus in saliva [29].

Previous studies have demonstrated that salivary specimens have a higher than $90 \%$ concordance rate with nasopharyngeal specimens in the detection of respiratory viruses [14].

The use of oral swabs is presumably applicable in early detection. By collecting oral swabs and testing RNA in 15 COVID19 patients, Zhang et al. found that half of them (50\%) were SARS-CoV-2 RNA positive in oral swabs, four (26.7\%) had positive anal swabs, six (40\%) had positive blood test, and three (20\%) were serum positive [30]. Dynamic viral RNA presence in saliva compared with anal swabs were analyzed among 16 patients. Among all swab positive together, most of the positive result was from oral swabs at early stage, while more positive came from anal swabs at late stage of COVID-19, suggesting that oral swabs may indicate early infection of SARS-CoV-2 but cannot be used as a discharge criteria $[23,30]$.

On the other hand, and to rule out contamination of respiratory secretion, Chen et al. collected saliva directly from the opening of salivary gland and found SARS-CoV-2 nucleic acid, which suggest the infection of salivary glands by SARSCoV-2. Thirteen cases who were nucleic acid positive by oropharyngeal swab among 31 COVID-19 patients were included, and four of them (12.90\%) were positive in saliva. Three cases of these four were critically ill patients in need of ventilator support, suggesting SARS-CoV-2 nucleic acid positive in salivary-gland-originated saliva as an indicator of severity of COVID-19 [23, 31].

Close contact between healthcare workers and patients is required in the collection of these specimen types, which compromise a risk of transmission of the virus to the healthcare workers. In addition, the collection of nasopharyngeal or oropharyngeal specimens causes discomfort and may cause bleeding, particularly in patients with condition such as thrombocytopenia [22, 32]. A recent clinical study indicates that 29\% of 138 hospitalized patients with COVID-19infected pneumonia in Wuhan, China, are healthcare workers [13].

Latest researches from RUCDR Infinite Biologics at Rutgers University have successfully validated saliva as being a viable bio sample source for SARS-CoV-2 detection when compared to nasopharyngeal or oropharyngeal swabs. According to them, the use of saliva to extract viral RNA was in fact a robust source for SARS-CoV-2 detection and equals in performance to the approved swab-based collection samples [22, 33].

However, it seems that the diagnostic value of saliva depends on how saliva specimens are harvested. Saliva from deep throat (91.67 and $86.96 \%$ corresponding to two studies), from oral cavity (50\%), or from salivary glands (12.90\%) indicates a diagnostic tendency of decreased positive rate of SARS-CoV-2 RNA among COVID-19 patients [14, 15, 30, 31, 34].

Various sizes from salivary droplets generated by breathing, talking, and sneezing, large droplets easily fall into the floor and only set up short-distance transmission. Saliva could form aerosols and reach a distant host along airflow when in a favorable environment. So far, no solid evidence supports that SARS-CoV or SARS-CoV-2 can survive in air outdoors for long time to set up long-distance aerosol transmission [35]. Hence, wearing masks to prevent formation of infectious saliva droplets projecting to the air, thorough disinfection of indoor air to block dissemination of infectious saliva droplets, and keep a distance with people not to acquire infectious saliva droplets could slow down COVID-19 epidemic to a certain degree [23].

In conclusion, although saliva is currently perceived as an enemy in the battle against COVID-19 due to it being a prominent source for disease transmission via droplets and possibly aerosols, it is also apparent that it can be harnessed as a friend in the detection of the virus and an individual's immunity to it. Early diagnosis of SARS-CoV-2 is still difficult and the diagnostic value of saliva specimens for SARS-CoV-2 nucleic acid examination remains limited but promising. Specific guidelines are needed to standardize the method for collection of salivary specimens, and implement the use of appropriate assays, and processing methods, which is crucial to improve effective strategies for prevention, especially for dentists and healthcare professionals that perform aerosol-generating procedures. 


\subsection{Gustatory disorders: Hypogeusia and ageusia}

Viral Upper Respiratory Tract Infections can lead to olfactory and gustatory Disorders of varying degrees and duration, which can also occur in $70 \%$ of cases caused by rhinovirus, influenza virus and parainfluenza, respiratory syncytial virus, adenovirus and the severe acute respiratory syndrome virus (SARS-CoV-2). Several observations indicate that gustatory and olfactory disorders were also added to the list of symptoms of coronavirus infection that should warn people to self-isolate before medical consultation. This topic deals only the data concerning the taste disorders [15, 18, 36].

The meta-analysis conducted by Tong JY et al [36], using a random-effects model, demonstrated a significant prevalence of gustatory dysfunction among patients with COVID-19. Analysis of nine studies with 1390 COVID-19 patients showed that 626 total patients reported some level of gustatory dysfunction, which demonstrated $43.93 \%$ (95\% CI, 20.46\%$68.95 \%$ ) prevalence of this dysfunction among these patients.

Gustatory dysfunction may also represent an early suggestive symptom of SARS-CoV-2 infection, but this symptom appears to have been less robustly studied. As a result, it remains unclear as to whether gustatory dysfunction represents a distinct clinical manifestation of the virus or if this occurs secondary to olfactory dysfunction [36].

The pathogenesis of this chemo sensitive disorder has not yet been clarified. Moreover, the comprehension of this mechanism could be useful to understand the way in which the virus spreads through the organism [37].

Angiotensin-converting enzyme 2 (ACE2) is the cellular receptor for SARS-CoV-2 [28]. ACE2 receptors are expressed on the mucous membrane of the whole oral cavity, especially on the tongue [38]. The role of ACE2 in modulating taste perception has been highlighted in many studies analyzing the chemo sensitive side effects of ACE2 inhibitors and angiotensin II blockers. The mechanism by which ACE2 inhibitors cause taste disturbance is unclear but does not appear to be related to any alteration in serum and saliva zinc levels. Probably, these drugs inactivate the G-protein-coupled proteins and sodium-ion channels present in the taste receptors. The taste disturbance generally regresses after the suspension of treatment [37-40].

Moreover, the Middle East Respiratory Syndrome (MERS) coronavirus may bind to the sialic acid receptors, an ability which has also recently been described for SARS-CoV-2 [41, 42]. Sialic acid is a fundamental component of the salivary mucin, and it protects the glycoproteins that convey gustatory molecules inside the taste pores from premature enzymatic degradation [43]. A reduction of sialic acid in the saliva is associated with an increase in the gustatory threshold [44]. In such a way, SARS-CoV-2 could therefore occupy the binding sites of sialic acid on the taste buds, accelerating the degradation of the gustatory particles. Another possibility is that the ability to perceive flavors in these patients is adversely affected by the concomitant presence of olfactory disturbances, due to the intimate functional correlation between these two chemosensory systems [37].

As well as heighten vigilance for viral spread, an increased awareness of this fact may encourage earlier diagnosis and treatment of COVID-19. The studies have shown that both olfactory and gustatory disorders of varying intensities and in which onset occurred prior to the general symptoms of SARS-CoV-2 infection may occur, and such disorders should be considered as part of the symptoms that may be present, even in mild cases of COVID19. There is still no scientific evidence of specific treatments for such disorders in COVID-19.

\subsection{Oral dermatologic lesions}

Other oral manifestations have been observed in patients with COVID-19. Nevertheless, there is still a question if these lesions are due to coronavirus infection or secondary manifestations resulting from the patient's systemic condition.

There have been some COVID-19 cases reporting painful oral manifestations including lesions like irregular oral ulcer of the tongue [45], white lingual plaque [46], oral vesiculobullous lesion; as seen in herpes simplex lesions and erythema multiform, were also observed in single cases $[47,48]$.

Pathogenesis of oral lesions could be associated with a variable inflammatory reaction of COVID-19 that can induce vascular inflammation [49]. Thus, these oral lesions could be an inaugural symptom of Covid-19, which needs to be proven in larger cohorts of patients.

Presence of these oral conditions support the hypothesis that they are suggestive of secondary lesions resulting from the deterioration of systemic health or due to treatments for COVID-19 [46]. 
The epidemiologic significance of the oral manifestations remains unclear, thus necessitating larger observational studies to reveal the prevalence and the onset of these symptoms. Healthcare workers such as dentists, dermatologists other medical doctors must be encouraged to perform intraoral examinations in patients suspected or affected by SARS$\mathrm{CoV}-2$ always when having the recommended protection measures available.

\subsection{COVID-19 treatment and oral health}

Medical treatment of COVID-19 patients causes side effects; nevertheless, their benefits exceed the disadvantages. Because of intense pharmacotherapy, some of patients may suffer from oral problems associated with soft tissues, saliva production and neurological-based oral sensations, even after recovery from COVID-19 [46, 50].

The experimental anti-viral treatment using lopinavir and ritonavir may be responsible for side effects (<2\%) affecting oral cavity among the other parts of gastrointestinal track, such as stomatitis, mouth ulcers and dry mouth [50]. It has been reported that interferon alfa/beta, well known for their anti-viral activity, could be responsible of dry mouth, which can result in frequent cases of oral thrush [50].

At present, when facing the reality of lack of data about the relationship between SARS-CoV-2 and oral diseases, it could be easy to imagine that a vast proportion of COVID-19 symptomatic and intensely treated patients must develop some sort of oral complications. If the patient survives, especially during recovery phase, it is likely that will need an adjunct antifungal and/or antimicrobial treatment, supported by cytoprotective topical measures.

The use of systemic and topical steroids is not be suitable in COVID-19 infection [51], some of immune related longterm oral medicine conditions (pemphigus, lichen planus, pemphigoid) may potentially exacerbate in SARS-CoV-2 positive patients who were advised to discontinue such therapy [50,52].

As a consequence of life-saving therapies, such as external ventilation and blood oxygenation, in severely ill hospitalized patients, deterioration of oral health had been detected, especially in case of those staying in intensive care units. A lack of oral care as treatment priority is given to advanced medical care; intubation, tracheostomy, external ventilation, as well as mouth breathing, hyposalivation can lead to rapid oral health deterioration and subsequent complications, affecting also the lower respiratory track, similar to aspiration pneumonia [50,53]. Disturbances of oral microbiota balance are a result of systemic treatments and intraoral ecosystem alteration may lead to further problems.

Severe COVID-19 acute infection, along with associated therapeutic measures, could potentially contribute to negative outcomes with regard to oral health, likely leading to various opportunistic fungal infections, xerostomia linked to decreased salivary flow, ulcerations and gingivitis because of impaired immune system and/or susceptible oral mucosa. It is worth noting that cytokine storm caused by dysregulated humoral and cellular mechanisms can aggravate existing autoimmune conditions within the oropharyngeal area.

Patients recovering from COVID-19 disease need more post-acute care to recuperate from primary and concomitant infection, with a necessary monitoring of their oral health, especially during transition from hospital to other care settings and homes. The reinforcement of existing oral medicine facilities should also allow to continue the provision of secondary care for patients with existing oral diseases, regardless their COVID-19 negative/positive status, whose follow-up treatment was postponed due to emergency measures introduced.

\section{Conclusion}

In conclusion, COVID-19 pandemic has required medical professionals to make new decisions with development of innovative methods of care that focused on patient safety, attenuation efforts, and clinical management of disease processes.

Oral cavity could be responsible of transmission of this virus, and dental professionals that perform aerosol-generating procedures, are at high risk for nosocomial infection and can become potential carriers of the disease, which is crucial to improve effective strategies for prevention.

Specific general measures comprise daily monitoring of the temperature and testing the health care provider team; use of N95 masks; distance from the workplace (when possible) with the establishment of network technologies to communicate with patients; social distance; mobility restriction; avoid crowd places; diagnostic tests and isolation of infected individuals as well as their families. Particularly for dentists it is important to follow guidance protocols and 
new tools/technologies for dental practice aimed at safeguarding oral health professionals, as well as the population under their care.

This review may provide new clinical information to raise awareness among dental practitioners that careful intraoral examination is mandatory and could increase the ability to diagnose COVID-19 patients sooner. The Sars-CoV-2 infection is responsible for several events in the mouth. More studies, however, are necessary to understand the real connection between oral cavity and COVID-19, and to demonstrate the previously exposed aspects in SARS-CoV-2 positive patients.

\section{Compliance with ethical standards}

\section{Disclosure of conflict of interest}

There is no conflict of interest

\section{References}

[1] The Lancet. Emerging understandings of 2019-nCov. Lancet 2020; 395: 311.

[2] Wu F, Zhao S, Yu B, et al. A new coronavirus associated with human respiratory disease in China. Nature. 2020; 579(7798): 265-269.

[3] Sohrabi C, Alsafi Z, O'Neill N, et al. World Health Organization declares global emergency: a review of the 2019 novel coronavirus (COVID-19). Int J Surg. 2020; 76: 71-6.

[4] WHO director-general's opening remarks at the media briefing on COVID-19. 3 March 2020.

[5] World Health Organization. WHO Director-General's remarks at the media briefing on $2019 \mathrm{nCoV}$ on. 11 February 2020.

[6] Doremalen N, Bushmaker T, Morris DH, Holbrook MG, Gamble A, Williamson BN, et al. Aerosol and surface stability of SARS-CoV-2 as compared with SARS-CoV-1. N Engl J Med. 2020 Mar;NEJMc2004973

[7] Marra MA, Jones SJM, Astell CR, Holt RA, Brooks-Wilson A, Butterfield YSN, et al. The genome sequence of the SARS-associated coronavirus. Science. 2003 May; 300(5624): 1399-404.

[8] Pereira LJ, Pereira CV, Murata RM, Pardi V, Pereira-Dourado SM. Biological and social aspects of Coronavirus Disease 2019 (COVID-19) related to oral health. Braz Oral Res. 2020; 34: e041.

[9] Wolff MH, Sattar SA, Adegbunrin O, Tetro J. Environmental survival and microbicide inactivation of coronaviruses. Coronaviruses with Special Emphasis on First Insights Concerning SARS. 2005; 201-212.

[10] Abbag HF, El-Mekki AA, Al Bshabshe AA, Mahfouz AA, Al-Dosry AA, Mirdad RT, et al. Knowledge and attitude towards the Middle East respiratory syndrome coronavirus among healthcare personnel in the southern region of Saudi Arabia. J Infect 2018; 11(5): 720-722.

[11] Chen N, Zhou M, Dong X, Qu J, Gong F, Han Y, Qiu Y, Wang J, Liu Y, Wei Y, et al. Epidemiological and clinical characteristics of 99 cases of 2019 novel coronavirus pneumonia in Wuhan, China: a descriptive study. Lancet. 2020; 395(10223): 507-513.

[12] Guan W-J, Ni Z-Y, Hu Y, Liang W-H, Ou C-Q, He J-X, Liu L, Shan H, Lei C-L, Hui DS, et al. Clinical characteristics of 2019 novel coronavirus infection in China. medRxiv. 2020.

[13] Wang D, Hu B, Hu C, et al. Clinical characteristics of 138 hospitalized patients with 2019 novel coronavirusinfected pneumonia in Wuhan, China. JAMA. 2020; 323(11): 1061- 1069.

[14] To KK, Tsang OT, Chik-Yan Yip C et al. Consistent detection of 2019 novel coronavirus in saliva. Clin Infect Dis. [published online ahead of print. 12 Feb 2020.

[15] Sabino-Silva R, Jardim ACG, Siqueira WL. Coronavirus COVID-19 impacts to dentistry and potential salivary diagnosis. Clin Oral Investig. 2020; 24(4): 1619-1621.

[16] Xie X, Li Y, Sun H, Liu L. Exhaled droplets due to talking and coughing. J R Soc Interface. 2009; 6(6): S703-S714. 
[17] Chen Y, Liu Q, Guo D. Emerging coronaviruses: genome structure, replication, and pathogenesis. J Med Virol. 2020; 92(4): 418-423.

[18] Corman VM, Landt O, Kaiser M, et al. Detection of 2019 novel coronavirus (2019-nCoV) by real-time RT-PCR. Euro Surveill. 2020; 25(3): 2000045.

[19] Pfaffe T, Cooper-White J, Beyerlein P, Kostner K, Punyadeera C: Diagnostic potential of saliva: current state and future applications. Clin Chem. 2011; 57: 675-687.

[20] Lee JM, Garon E, Wong DT: Salivary diagnostics. Orthod Craniofac Res. 2009; 12: 206-211.

[21] Mittal S, Bansal V, Garg S, Atreja G, Bansal S: The diagnostic role of saliva-a review. J Clin Exp Dent. 2011; 3: $314-$ 320.

[22] Sri Santosh T, Parmar R, Anand H, Srikanth K, Saritha M. A Review of Salivary Diagnostics and Its Potential Implication in Detection of Covid-19. Cureus 12(4): e7708.

[23] Xu R, Cui B, Duan X, Zhang P, Zhou X, Yuan Q. Saliva: potential diagnostic value and transmission of 2019-nCoV. Int J Oral Sci. 2020; 12(1): 11.

[24] Barzon L, Pacenti M, Berto A, Sinigaglia A, Franchin E, Lavezzo E, Brugnaro P, Palù G (2016) Isolation of infectious Zika virus from saliva and prolonged viral RNA shedding in a traveller returning from the Dominican Republic to Italy. Euro Surveill. 2016;21(10):30159

[25] Zuanazzi D, Arts EJ, Jorge PK, Mulyar Y, Gibson R, Xiao Y, Bringel Dos Santos M, Machado MAAM, Siqueira WL. Postnatal identification of zika virus peptides from saliva. J Dent Res. 2017; 96(10):1078- 1084.

[26] Segal A, Wong DT. Salivary diagnostics: enhancing disease detection and making medicine better. Eur J Dent Educ. 2008; 12(1): 22-29.

[27] Zhu N, Zhang D, Wang W, et al. A Novel Coronavirus from Patients with Pneumonia in China, 2019. N Engl J Med. 2020; 382(8): 727-733.

[28] Zhou P, Yang XL, Wang XG, et al. A pneumonia outbreak associated with a new coronavirus of probable bat origin. Nature. 2020; 579(7798): 270-273.

[29] Liu L, Wei Q, Alvarez X, et al. Epithelial cells lining salivary gland ducts are early target cells of severe acute respiratory syndrome coronavirus infection in the upper respiratory tracts of rhesus macaques. J Virol. 2011; 85(8): 4025-4030.

[30] Zhang W, Du RH, Li B, et al. Molecular and serological investigation of 2019-nCoV infected patients: implication of multiple shedding routes. Emerg Microbes Infect. 2020; 9(1): 386-389.

[31] Chen Lili, Zhao Jiajia, Peng Jinfeng, Li Xiaoshuang, Deng Xuliang, Geng Zhi. Detection of 2019-nCoV in saliva and characterization of oral symptoms in COVID-19 patients. SSRN Electron J. 31 Mar 2020.

[32] Chan JF, Yuan S, Kok KH, et al. A familial cluster of pneumonia associated with the 2019 novel coronavirus indicating person-to-person transmission: a study of a family cluster. Lancet. 2020; 395: 514-523.

[33] Rutgers launches genetic testing service for new coronavirus. [Apr;2020]; https://www.rutgers.edu/news/rutgers-launches-genetic-testing-service-ne... 2020

[34] To KK, Tsang OT, Leung WS, et al. Temporal profiles of viral load in posterior oropharyngeal saliva samples and serum antibody responses during infection by SARS-CoV-2: an observational cohort study. Lancet Infect Dis. 2020; 20(5): 565-574.

[35] Tang JW, Li Y, Eames I, Chan PK, Ridgway GL. Factors involved in the aerosol transmission of infection and control of ventilation in healthcare premises. J. Hosp. Infect. 2006; 64: 100-114.

[36] Tong JY, Wong A, Zhu D, Fastenberg JH, Tham T. The Prevalence of Olfactory and Gustatory Dysfunction in COVID19 Patients: A Systematic Review and Meta-analysis. Otolaryngol Head Neck Surg. 2020; 163(1): 3-11.

[37] Vaira LA, Salzano G, Fois AG, Piombino P, De Riu G. Potential pathogenesis of ageusia and anosmia in COVID-19 patients [published online ahead of print. 7 Apr 2020.

[38] $\mathrm{Xu} \mathrm{H}$, Zhong L, Deng $\mathrm{J}$ et al. High expression of ACE2 receptor of 20119-nCoV on the epithelial cells of oral mucosa. Int J Oral Sci. 2020; 12: 8. 
[39] Tsuruoka S, Wakaumi M, Araki N, Ioka T, Sugimoto K, Fujimura A. Comparative study of taste dirsurbance by losartan and perindopril in healthy volunteers. J Clin Pharmacol. $2005 ; 45-1319-23$.

[40] Suliburska J, Duda G, Pupek-Musialik D. The influence of hypotensive drugs on the taste sensitivity in patients with primary hypertension. Acta Pol Pharm. 2012; 60: 121-7.

[41] Park YJ, Walls AC, Wang Z et al. Structures of MERS-CoV spike glycoprotein in complex with sialoside attachment receptors. Nat Struct Mol Biol. 2019; 26: 1151-7.

[42] Milanetti M, Miotto M, Di Rienzo L, Monti M, Gosti G, Ruocco G. In-Silico evidence for two receptors based strategy of SARS-CoV-2. BioRyX 2020.03.24.006197; doi: https://doi.org/10.1101/2020.03.24.006197.

[43] Witt M, Miller Jr IJ. Comparative lectin histochemistry on taste bud in foliate, circumvallate and fungigorm papillae of the rabbit tongue. Histocemistry. 1992; 98(3): 173-82.

[44] Pushpass RG, Pellicciotta N, Kelly C, Proctor G, Carpenter GH. Reduced salivary mucin binding and glycosylation in older adults influences taste in an in vitro cell model. Nutrients. 2019; 11: 228.

[45] Chaux-Bodard AG, Deneuve S, Desoutter A. Oral manifestation of Covid-19 as an inaugural symptom? Journal of Oral Medicine and Oral Surgery. 2020; 26(2): 18.

[46] Dos Santos JA, Costa Normando AG, Da Silva RLC, De Paula RM, Cembranel AC, Santos-Silva AL, Silva Guerra EN. Oral mucosal lesions in a COVID-19 patient: new signs or secondary manifestations. Int J Infect Dis. 2020; 97: 326-328.

[47] Martín Carreras-Presas C, Amaro Sánchez J, López-Sánchez AF, Jané-Salas E, Somacarrera Pérez ML. Oral vesiculobullous lesions associated with SARS-CoV-2 infection [published online ahead of print. 5 May 2020.

[48] Recalcati S. Cutaneous manifestations in COVID-19: a first perspective. J Eur Acad Dermatol Venereol 2020; 34 : e212-13.

[49] Madjid M, Safavi-Naeini P, Solomon SD, Vardeny O. Potential Effects of Coronaviruses on the Cardiovascular System: A Review. JAMA Cardiol. Published online. 20 March 2020.

[50] Dziedzic A, Wojtyczka R. The impact of coronavirus infectious disease 19 (COVID-19) on oral health [published online ahead of print. 18 Apr 2020.

[51] Mehta P, McAuley DF, Brown M, et al. COVID-19: consider cytokine storm syndromes and immunosuppression. Lancet. 2020; 395(10229): 1033-1034.

[52] Yuen KS, Ye ZW, Fung SY, Chan CP, Jin DY. SARS-CoV-2 and COVID-19: The most important research questions. Cell Biosci. 2020; 10: 40.

[53] Wu C, Chen X, Cai Y, et al. Risk Factors Associated with Acute Respiratory Distress Syndrome and Death in Patients With Coronavirus Disease 2019 Pneumonia in Wuhan, China [published online ahead of print. 13 Mar 2020. 